\title{
Die Bestimmung des Stellvertreters des Ministerpräsidenten als Teil der Regierungsbildung - Allgemeine Grundsätze und verfassungsrechtliche Besonderheiten in Rheinland-Pfalz
}

\author{
Richard Ley
}

Die Frage, ob die bisherige Stellvertreterin des rheinland-pfälzischen Ministerpräsidenten, Eveline Lemke, auch unter der am 16. Januar 2013 neu gewählten Ministerpräsidentin Malu Dreyer diese Funktion ausüben könne, wurde öffentlich kontrovers diskutiert. Während der Autor in der FAZ ${ }^{1}$ die seines Erachtens notwendige, jedoch nicht durchgeführte Neubestimmung Lemkes zur Stellvertreterin als „Panne“ bezeichnete, stellte der Vorsitzende der rheinland-pfälzischen FDP die Frage ${ }^{2}$, ob das Land nicht auf eine „Verfassungskrise“ zusteuere, wenn die stellvertretende Ministerpräsidentin eventuell ein Gesetz unterzeichne, wozu sie nicht die „Amts-Legitimation“ habe, und forderte deshalb ein ausführliches externes Gutachten. Landtagspräsident Joachim Mertes hatte sich bereits vorher auf eine Stellungnahme des wissenschaftlichen Dienstes des Landtages berufen ${ }^{3}$ und unter anderem ausgeführt, dass nach dem 1991 eingefügten Art. 98 Abs. 3 LV ein Minister das Amt als Vize-Ministerpräsident nur durch Entlassung oder Entzug verliere. ${ }^{4}$ In der Regionalpresse wurde in Einspaltern über die Kontroverse berichtet ${ }^{5}$; die verfassungsrechtliche Problematik geriet vor allem durch den Artikel des Autors „Regierungswechsel in Rheinland-Pfalz während der Wahlperiode " 6 an die politische Öffentlichkeit.

\section{Bedeutung sowie Standort der Regelungen über die Bestimmung des Stellvertreters des Ministerpräsidenten ${ }^{7}$}

Regelungen über die Stellvertretung sollen gewährleisten, dass auch im Falle der Verhinderung des Ministerpräsidenten alle Aufgaben des Regierungschefs wahrgenommen werden

1 Vgl. Richard Ley, Die Mainzer Panne, in: FAZ vom 27. Februar 2013, S. 30.

2 Vgl. „Verfassungskrise in Rheinland-Pfalz?“, Presseerklärung der FDP Rheinland-Pfalz vom 24. Mai 2013, http://www.fdp-rlp.de/Verfassungskrise-in-Rheinland-Pfalz/1157c1695i1p162/index. html (Abruf am 24. Mai 2013).

3 Vgl. „Eveline Lemke ist stellvertretende Ministerpräsidentin. Landtagspräsident Mertes erklärt, dass die Verfassungslage eindeutig ist", Presseinformation des Landtages Rheinland-Pfalz vom 24. Mai 2013, http://www.landtag.rlp.de/icc/Internet-DE/sub/499/49910067-b6f1-dd31-41c2004586d35f8f,,, aaaaaaaa-aaaa-aaaa-bbbb-000000000014\&page=2\&pagesize=10.htm (Abruf am 3. Juni 2013).

4 Die Stellungnahme des wissenschaftlichen Dienstes konnte nicht zugänglich gemacht werden.

5 Vgl. zum Beispiel die Berichterstattung in der Rheinzeitung im entsprechenden Zeitraum.

6 Vgl. Richard Ley, Regierungswechsel in Rheinland-Pfalz während der Wahlperiode, in: Zeitschrift für Landes- und Kommunalrecht Hessen, Rheinland-Pfalz, Saarland, 4. Jg. (2013), H. 4, S. 133 - 138.

7 Die Bezeichnung Ministerpräsident wird nachfolgend als Synonym auch für anderweitige Bezeichnungen des Landeschefs in Berlin (Regierender Bürgermeister), Bremen (Präsident des Senats) und Hamburg (Erste Bürgermeisterin beziehungsweise Erster Bürgermeister oder Präsidentin beziehungsweise Präsident des Senats) verwendet. Des Weiteren wird im Text in der Regel die männliche Formulierung verwendet, obwohl in den Bundesländern Hamburg, Niedersachsen und Schleswig-Holstein sowohl die weibliche als auch die männliche Bezeichnung in den Verfassungen benutzt werden. 
können und die Funktionsfähigkeit des wichtigsten Verfassungsorganes der Exekutive gewahrt bleibt. ${ }^{8}$ Im Handbuch des Staatsrechts wird die entsprechende bundesrechtliche Regelung (Art. 69 Abs. 1 GG) unter anderem auch im Kapitel „Funktionsstörungen in der Staatsorganisation“ behandelt und zwar in einem Abschnitt, in dem verschiedene „präventive Sicherungen der Funktionsfähigkeit" erörtert werden. ${ }^{\text {? }}$

Außer in den Bundesländern Brandenburg, Hessen und dem Saarland, wo die Stellvertreterfrage nur in den Geschäftsordnungen der Landesregierungen angesprochen wird, haben alle anderen Bundesländer diese Rechtsproblematik in den Landesverfassungen verankert. In fünf Ländern haben dabei die Landtage ein Mitwirkungsrecht und zwar entweder im Rahmen der gemeinsamen Bestätigung der Landesregierung beziehungsweise der Mitglieder der Landesregierung ${ }^{10}$ oder über ein Zustimmungserfordernis zur Bestimmung des Stellvertreters. ${ }^{11}$

\section{Pflicht des Ministerpräsidenten zur unverzüglichen Bestimmung seines Stellvertreters}

Zur Vermeidung von Funktionsstörungen hat der Ministerpräsident nicht nur das Recht, sondern nach einhelliger Literaturmeinung ${ }^{12}$ auch die Pflicht, seinen Stellvertreter zu bestimmen. ${ }^{13}$ Geht man von der Bedeutung der Bestellung eines Stellvertreters als Vorsorgemaßnahme für den nicht voraussehbaren Verhinderungsfall aus, so sollte die Bestellung unmittelbar im Zusammenhang mit der Regierungsbildung erfolgen. ${ }^{14}$ Bei einem Rücktritt des Stellvertreters bedeutet dies, dass die Bestellung eines Nachfolgers unverzüglich (das heißt ohne schuldhaftes Zögern) ${ }^{15}$ erfolgen sollte, damit „bei Eintritt des Verhinde-

8 Vgl. hierzu zum Beispiel Alfred Katz, in: Paul Feuchte (Hrsg.), Verfassung des Landes Baden-Württemberg, Kommentar, München 1987, Art. 46 Rn. 11; Karl Schweiger, in: Hans Nawiasky / ders. I Franz Knöpfle (Hrsg.), Die Verfassung des Freistaates Bayern, München 2006, Art. 46 Rn. 2.

9 Vgl. Eckart Klein, Funktionsstörungen in der Staatsorganisation, in: Josef Isensee / Paul Kirchhof (Hrsg.), Handbuch des Staatsrechts der Bundesrepublik Deutschland, Heidelberg 1992, Band VII, $\$ 168$, Rn. 28.

10 Vgl. Art. 46 Abs. 3 LV-BW; Art. 101 Abs. 4 LV-HH und Art. 29 Abs. 3 LV-NS.

11 Vgl. Art. 46 LV-BY und Art. 105 Abs. 3 Satz 3 LV-RP.

12 Vgl. unter anderem Alfred Katz, a.a.O. (Fn. 8), Art. 46 Rn. 11; Heinzgeorg Neumann, Die Niedersächsische Verfassung, Handkommentar, Stuttgart u.a. 2000, Art. 29 Rn. 20; Lothar Hagebölling, Niedersächsische Verfassung, Kommentar, Wiesbaden 1996, Art. 29 Anm. 3; Christian Dästner, Die Verfassung des Landes Nordrhein-Westfalen, Kommentar, Stuttgart 2002, Art. 52 Rn. 6; Martin Nolte, in: Johannes Caspar / Wolfgang Ewer / ders. / Hans-Jochen Waack (Hrsg.), Verfassung des Landes Schleswig-Holstein, Kommentar, Kiel 2006, Art. 26 Rn. 25.

13 Dies ist auch hinsichtlich des Stellvertreters des Bundeskanzlers nach Art. 69 Abs. 1 GG allgemeine Ansicht; vgl. statt vieler zum Beispiel Klaus Stern, Das Staatsrecht der Bundesrepublik Deutschland, München 1980, Band II, $\$ 31$ II 4 b); Roman Herzog, in: Theodor Maunz / Günter Dürig (Hrsg.), Grundgesetz-Kommentar, München 2008, Art. 69 Rn. 4; Georg Hermes, in: Horst Dreier (Hrsg.), Grundgesetz-Kommentar, Tübingen 2006, Art. 69 Rn. 3, Rn. 4, Rn. 10.

14 Vgl. zum Beispiel Volker Epping, in: Hermann von Mangoldt / Friedrich Klein / Christian Starck (Hrsg.), Das Bonner Grundgesetz-Kommentar, München 2005, Art. 69 Rn. 5; Roman Herzog, a.a.O. (Fn. 13), Art. 69 Rn. 3.

15 Vgl. unter anderem Joachim Linck, in: ders. / Siegfried Jutzi / Jörg Hopfe, Die Verfassung des Freistaates Thüringen - Kommentar, Stuttgart u.a. 1994, Art. 70 Rn. 22; Volker Epping, in: ders. I Hermann Butzer / Frauke Brosius-Gersdorf I Ulrich Haltern / Veith Mehde / Kay Waechter (Hrsg.), Hannoverscher Kommentar zur Niedersächsischen Verfassung, Baden-Baden 2012, Art. 29 Rn. 
rungsfalls der Fortgang der Regierungsgeschäfte auf der Ministerpräsidentenebene "16 gesichert ist.

\section{Besonderheiten der rheinland-pfälzischen Verfassung bei der Konstituierung der Landesregierung}

Die Rechtslage hinsichtlich der Regierungsbildung unterscheidet sich in Rheinland-Pfalz auf den ersten Blick kaum von jener der anderen Bundesländer. Auf den zweiten Blick bestehen aber drei Unterschiede beziehungsweise Besonderheiten, die Auswirkungen auf einzelne Schritte der Bestellung der Landesregierung - unter anderem auch auf die Bestimmung des Stellvertreters des Ministerpräsidenten - nach der Neuwahl eines Ministerpräsidenten während der Wahlperiode haben.

Die erste Besonderheit ist das Fehlen einer Vorschrift vergleichbar Art. 69 Abs. 2 GG, die nahezu alle anderen Bundesländer kennen. ${ }^{17}$ Danach endet unter anderem mit einem Rücktritt des Regierungschefs auch die Amtszeit der anderen Mitglieder der Regierung. ${ }^{18}$ Diese fehlende Regelung führte bei drei der sechs Regierungswechsel während der Wahlperiode in Rheinland-Pfalz dazu, dass die meisten oder sogar alle Minister aus dem Kabinett des Vorgängers - ohne rechtliche Unterbrechung beziehungsweise Neuernennung - auch Mitglied des Kabinetts des neu gewählten Ministerpräsidenten wurden. ${ }^{19}$

Weitere Besonderheiten sind die Mitwirkungsrechte des Landtags bei der Regierungsbildung, die viele andere Landesverfassungen in diesem Ausmaß nicht kennen. So muss der Landtag durch ausdrückliche Beschlüsse die neu ernannten Minister bestätigen (Art. 98 Abs. 2 Satz 3 LV-RP) ${ }^{20}$ und dem vom Ministerpräsidenten bestimmten Stellvertreter zustimmen (Art. 105 Abs. 2 Satz 3 LV-RP). ${ }^{21}$ Ohne diese Beschlüsse können die neu ernannten Minis-

35. Dies ist auch die Ansicht der Literatur für Art. 69 Abs. 1 GG; vgl. zum Beispiel Roman Herzog, a.a.O. (Fn. 13), Art. 69 Rn. 10.

16 So Joachim Linck, a.a.O. (Fn. 15), Art. 70 Rn. 22.

17 Vgl. zum Beispiel Art. 55 Abs. 2 LV-BW; Art. 85 Abs. 1 LV-Bbg; Art. 113 Abs. 1, 2 LV-HS; Art. 50 Abs. 1 LV-MV; Art. 33 Abs. 2, 3 LV-NS; Art. 62 Abs. 2 NRW; Art. 87 Abs. 3 LV-SL; Art. 68 Abs. 2 LV-Sachs; Art. 71 Abs. 1 LV-SAnh; Art. 27 Abs. 1 LV-SH; Art. 75 Abs. 2 LV-TH.

18 Das Fehlen einer Art. 69 Abs. 2 GG entsprechenden Vorschrift in Rheinland-Pfalz hat auch Auswirkungen auf die Amtsdauer des Ministerpräsidenten und der nicht unbedingt erforderlichen Wahl des Ministerpräsidenten am Anfang einer Wahlperiode. Es besteht in Rheinland-Pfalz - wie zum Beispiel auch in Berlin - keine Koppelung von Amtszeit und Wahlperiode. Vgl. dazu ausführlich die herrschende Lehre bei Richard Ley, Die Wahl der Ministerpräsidenten in den Bundesländern. Rechtslage und Staatspraxis, in: ZParl, 41. Jg. (2010), H. 2, S. 390 - 420, S. 399; ders., Wahlen und Amtszeit der rheinland-pfälzischen Ministerpräsidenten, in: Zeitschrift für Landesund Kommunalrecht Hessen, Rheinland-Pfalz, Saarland, 1. Jg. (2007), S. 169 - 174, S. 172 f. m.w.N. Anderer Auffassung Siegfried Jutzi, Staatsrecht, in: Reinhard Hendler / Friedhelm Hufen / ders. (Hrsg.), Landesrecht Rheinland-Pfalz, Baden-Baden 2012, \$1 Rn. 78/79.

19 Vgl. dazu ausführlich Richard Ley, a.a.O. (Fn. 6), S. 135 f.

20 Regelungen über die Bestätigung aller oder einzelnen Mitglieder der Landesregierungen gibt es nur in fünf weiteren Bundesländern; vgl. Art. 46 Abs. 3 LV-BW; Art. 45 LV-BY; Art. 24 Abs. 2 LV-HH; Art. 101 Abs. 4 LV-HE und Art. 29 Abs. 3 LV-NS. Ein Sonderfall ist Bremen; dort werden nach Art. 107 Abs. 2 alle Mitglieder des Senats von der Bürgerschaft gewählt.

21 Eine vergleichbare Regelung gibt es nur in Bayern (Art. 46). In allen anderen Bundesländern hat das Landesparlament keinen Einfluss auf die Bestimmung des Stellvertreters. 
ter nicht ihre Amtsgeschäfte wahrnehmen, und der vom Ministerpräsidenten bestimmte Stellvertreter kann zum Beispiel bei Krankheit oder Abwesenheit des Ministerpräsidenten dessen Aufgaben und Funktionen nicht übernehmen. Der rheinland-pfälzische Bundesverfassungsrichter Egon Schunck hat deshalb in einer ersten Darstellung der Verfassung des Landes kritisch angemerkt, dass die „Landesregierung ... in ungewöhnlich weitgehendem, über das Notwendige hinausgehenden Maße vom Landtag abhängig"22 ist.

Die dritte Besonderheit betrifft den Standort der Regelung über die Bestimmung des Stellvertreters des Ministerpräsidenten. Unstrittig ist in der Literatur, dass die Bestimmung des Stellvertreters Teil der Regierungsbildung ist. ${ }^{23}$ Deshalb darf in den Ländern, in denen eine Bestätigung der Landesregierung durch den Landtag erforderlich ist, diese erst nach der Bestimmung des Stellvertreters erfolgen. ${ }^{24}$ Außer in Rheinland-Pfalz und Bayern findet sich dieser dritte Akt der Regierungsbildung auch im entsprechenden Artikel, in dem die anderen Schritte der Regierungsbildung - Wahl des Ministerpräsidenten und Ernennung der Minister - geregelt sind. ${ }^{25}$ Allein in Rheinland-Pfalz ist dieser Zusammenhang mit der Regierungsbildung nicht sogleich erkennbar. Die Ernennung und Entlassung der Minister ist, wie auch die Wahl des Ministerpräsidenten, in Art. 98 LV-RP geregelt, die Stellvertretung des Ministerpräsidenten aber in Art. 105 LV-RP, der unter anderem Vorschriften über den Vorsitz in der Landesregierung, die Geschäftsverteilung und das Kollegialprinzip enthält.

\section{Die Bestimmung des Stellvertreters durch die Ministerpräsidenten}

Im Gegensatz zu den Landesverfassungen von Mecklenburg-Vorpommern, Niedersachsen und Nordrhein-Westfalen, die ausdrücklich fordern, dass der Ministerpräsident (nur) ein Mitglied der Landesregierung, also einen Minister, mit der Wahrnehmung der Funktion des Stellvertreters beauftragen darf ${ }^{26}$, weisen die Landesverfassungen von Baden-Württemberg, Rheinland-Pfalz, Sachsen, Sachsen-Anhalt und Thüringen keinerlei einschränkende oder klarstellende Formulierungen auf. Sie führen nur aus, dass der Ministerpräsident „seinen Stellvertreter“ benennt. ${ }^{27}$ Nach der herrschenden Literaturmeinung kann dies „selbstverständlich “28 nur ein Mitglied der Landesregierung sein. ${ }^{29}$ Dies bedeutet unter anderem auch

22 Vgl. Egon Schunck, Die Verfassung für Rheinland-Pfalz vom 18. Mai 1947, in: Gerhard Leibholz (Hrsg.), Jahrbuch des Öffentlichen Rechts, Band 5, Tübingen 1956, S. 159 - 202, S. 177.

23 Vgl. unter anderem Klaus Braun, Kommentar des Landes Baden-Württemberg, Stuttgart u.a. 1984, Art. 46 Rn. 27; Heinzgeorg Neumann, Die Verfassung der Freien Hansestadt Bremen-Kommentar, Stuttgart u.a. 1996, Art. 113 Rn. 3; Volker Epping, a.a.O. (Fn. 15), Art. 29 Rn. 35; Lothar Hagebölling, a.a.O. (Fn. 12), Art. 29 Anm. 4.

24 Vgl. zum Beispiel Volker Epping, a.a.O. (Fn. 15), Art. 29 Rn. 35; Lothar Hagebölling a.a.O. (Fn. 12), Art. 29 Anm. 4.

25 Die Bayerische Verfassung behandelt die drei Schritte der Regierungsbildung - Wahl des Ministerpräsidenten, Berufung der Regierungsmitglieder und Bestimmung des Stellvertreters - in jeweils einem eigenen Artikel; vgl. Art. 44 bis 46 LV-BY.

26 Vgl. Art. 43 Satz 2 LV-MV; Art. 29 Abs. 2 LV-NS; Art. 52 Abs. 3 LV-NRW.

27 Vgl. Art. 46 Abs. 2 Satz 2 LV-BW; Art. 105 Abs. 3 Satz 3 LV-RP; Art. 60 Abs. 4 Satz 2 LV-Sachs; Art. 65 Abs. 3 LV-SAnh.

28 So AdolfSüsterhenn / Hans Schäfer, Kommentar der Verfassung für Rheinland-Pfalz, Koblenz 1950, Art. 105 Anm. 3.

29 Vgl. unter anderem Alfred Katz, a.a.O. (Fn. 8), Art. 46 Rn. 11; Klaus Braun, a.a.O. (Fn. 23), Art. 46 Rn. 27; Rudolf Spreng / Willi Birn / Paul Feuchte, Die Verfassung des Landes Baden-Württem- 
für Rheinland-Pfalz, dass (nur) Minister diese Funktion wahrnehmen können, da die Landesregierung nach Art. 98 Abs. 1 LV-RP nur aus dem Ministerpräsidenten und den Ministern besteht. ${ }^{30}$

Diese Festlegung hat aber auch eine zeitliche Auswirkung. Die Bestimmung des Stellvertreters des Ministerpräsidenten kann erst nach der Ernennung der Minister erfolgen, das heißt, die Bestimmung des Stellvertreters des Ministerpräsidenten ist der letzte Akt der Regierungsbildung.

\section{Die verfassungsrechtliche Stellung als Minister und als Stellvertreter des Ministerpräsidenten}

In den Landesverfassungen werden die beiden Schritte der Regierungsbildung, Ernennung der Minister und Bestimmung des Stellvertreters des Ministerpräsidenten, jeweils in eigenen Sätzen, Halbsätzen, Absätzen oder Artikeln geregelt. Darüber hinaus werden für die beiden Akte aber auch verschiedene Begriffe verwendet, die ihren Grund in der unterschiedlichen rechtlichen Stellung der beiden Positionen haben.

\subsection{Die unterschiedlichen Begriffe für die beiden Staatsakte}

Die Mitglieder der Landesregierung werden vom Regierungschef berufen ${ }^{31}$ beziehungsweise ernannt. ${ }^{32}$ Für den Akt der Bestellung des Stellvertreters des Ministerpräsidenten werden in den Landesverfassungen und Geschäftsordnungen zumeist andere Begriffe verwendet: Es dominieren „bestimmen“33 und „bestellen“34. Nach den insoweit wortgleichen Verfassungen von Nordrhein-Westfalen und Mecklenburg-Vorpommern „beauftragt“ der Ministerpräsident ein Mitglied der Landesregierung mit seiner Vertretung. ${ }^{35}$ Die unterschiedlichen sprachlichen Begriffe - bestimmen / bestellen / beauftragen - erweisen sich als Synonym und führen zu keinerlei materiellen Auswirkungen auf den Staatsakt selbst.

berg, Kommentar, Stuttgart / Köln 1954, S. 176; Klaus-Eckart Gebauer, in: Christoph Grimm / Peter Caesar (Hrsg.), Verfassung für Rheinland-Pfalz. Kommentar, Baden-Baden 2001, Art. 105 Rn. 23; anderer Auffassung Andreas Reich, Verfassung des Landes Sachsen-Anhalt. Kommentar, Bad Honnef 1994, Art. 65 Rn. 7 - nach seiner Ansicht in der Regel nur ein Minister.

$30 \mathrm{Vgl}$. auch entsprechende Regelungen in den anderen Bundesländern wie zum Beispiel Art. $64 \mathrm{Abs}$. 1 Satz 2 LV-SAnh; Art. 70 Abs. 2 LV-TH.

31 Der Begriff Berufung wird in folgenden Verfassungen verwendet: Art. 46 Abs. 2 Satz 1 LV-BW; Art. 45 LV-BY; Art. 34 Abs. 2 Satz 1 LV-HH; Art. 29 Abs. 2 LV-NS; Art. 60 Abs. 4 Satz 1LV-Sachs; Art. 26 Abs. 2 Satz 2 LV-SH.

32 Der Begriff Ernennung wird in folgenden Verfassungen verwenden: Art. 56 Abs. 2 Satz 1 LV-B; Art. 84 LV-BBG; Art. 101 Abs. 2 Satz 1 LV-HE; Art. 43 Satz 1 LV-MV; Art. 52 Abs. 3 Satz 1 LV-NRW; Art. 98 Abs. 2 Satz 2 LV-RP; Art. 87 Abs. 1 Satz 2 LV-SL; Art. 65 Abs. 3 LV-SAnh; Art. 70 Abs. 4 Satz 1 LV-TH.

33 Vgl. Art. 46 LV-BY; Art. 29 Abs. 2 LV-NS; Art. 105 Abs. 2 Satz 3 LV-RP; Art. 70 Abs. 4 Satz 2 LV-TH.

34 Vgl. Art. 46 Abs. 2 Satz 2 LV-BW; $\$ 7$ Abs. 1 GeschO-LReg-BBG; $\$ 5$ Abs. 1 Satz 1 GeschOLReg-HE; $\$ 1$ Abs. 5 GeschO-LReg-SL; Art. 60 Abs. 4 Satz 2 LV-Sachs; Art. 26 Abs. 2 Satz2 LV-SH.

35 Vgl. Art. 43 Satz 2 LV-MV; Art. 52 Abs. 3 Satz 2 LV-NRW. 
In Berlin „ernennt“ der Regierende Bürgermeister zwei Senatoren „zu seinen Stellvertretern (Bürgermeister)“ (Art. 56 Abs. 2 Satz 2 LV-B) und in Hamburg „beruft“ nach Art. 34 Abs. 2 Satz 1 LV-HH die „Erste Bürgermeisterin oder der Erste Bürgermeister ... die Stellvertreterin (Zweite Bürgermeisterin) oder den Stellvertreter (Zweiter Bürgermeister)“. Allein in diesen beiden Stadtstaaten ${ }^{36}$ werden die Stellvertreter also ernannt beziehungsweise berufen. Es werden somit die Begriffe verwendet, die in den anderen Landesverfassungen und dem Grundgesetz für die Ernennung / Berufung der Mitglieder der Regierung gebraucht werden. Der Grund liegt in der unterschiedlichen rechtlichen Stellung der Stellvertreter in diesen beiden Ländern. Sie führen eigene Bezeichnungen und zwar Bürgermeister mit und ohne Zusatz und zeigen damit auch die Nähe zu kommunalverfassungsrechtlichen Regelungen.

\subsection{Das Ministeramt - ein öffentlich-rechtliches Amtsverhältnis}

Nach einhelliger Ansicht stehen die Personen, die vom Ministerpräsidenten zu Ministern ernannt beziehungsweise berufen werden und vor oder bei Amtsantritt ihren Eid ablegen, genauso wie der Ministerpräsident selbst in einem öffentlich-rechtlichen Amtsverhältnis. Dies kommt teilweise direkt ${ }^{37}$ oder indirekt in einzelnen Bestimmungen der Landesverfassungen oder in entsprechenden Landesgesetzen zum Ausdruck. Exemplarisch sollen hier auf die Bestimmungen über den „Amtseid“38, auf die teilweise erforderliche Bestätigung der Landesregierung oder der Minister vor „Amtsübernahme“39 oder das „Ende der Amtszeit“40 verwiesen werden. In den meisten Bundesländern gibt es auch so genannte Ministergesetze, die das Rechtsverhältnis der Mitglieder der Landesregierungen regeln. So wird in RheinlandPfalz $\$ 1$ MinG-RP ausdrücklich ausgeführt, dass die Mitglieder der Landesregierung in einem „öffentlich-rechtlichen Amtsverhältnis“ zum Land stehen. Nach $\$ 3$ MinG-RP erhalten die ernannten Minister eine „Urkunde über die Berufung“ beziehungsweise „Urkunde über die Beendigung des Amts“.

\subsection{Die Stellvertreterposition - kein Amt, sondern eine Funktionsstellvertretung}

Der Ministerpräsident überträgt nach einhelliger Literaturmeinung dem Stellvertreter jedoch kein weiteres Amt ${ }^{41}$, sondern nur eine Funktion. Im Gegensatz zum US-amerikanischen Vizepräsidenten haben die Stellvertreter der Regierungschefs im Bund und in den

36 Im dritten Stadtstaat, der Freien Hansestadt Bremen, wird der Stellvertreter des Präsidenten des Senats vom gesamten Senat gewählt; vgl. Art. 114; Art. 115 Abs. 1 LV-HB.

37 Nach Art. 33 Abs. 1 LV-SH und Art. 72 Abs. 1 LV-TH stehen die Mitglieder der Landesregierung in „einem besonderen öffentlich-rechtlichen Amtsverhältnis“.

38 In den Landesverfassungen heißt es unter anderem, die Minister leisten „beim Amtsantritt“ beziehungsweise „vor Amtsantritt“ den „Amtseid“. Vgl. die Belege unter anderem bei Richard Ley, Die Ministerpräsidenten in Hessen, Rheinland-Pfalz und dem Saarland, in: Zeitschrift für Landes- und Kommunalrecht Hessen, Rheinland-Pfalz, Saarland, 5. Jg. (2011), H. 10, S. 361 - 368, S. 361 f.; ders., a.a.O. (Fn. 6), S. $134 \mathrm{f}$.

39 Vgl. zum Beispiel die Regelungen in Art. 46 Abs. 3 LV-BW; Art. 29 Abs. 3 LV-NS.

40 Vgl. zum Beispiel Art. 55 Abs. 2 LV-BW; Art. 85 LV-BBG; Art. 87 Abs. 3 LV-SL.

41 Vgl. zum Beispiel Klaus-Eckart Gebauer, a.a.O. (Fn. 29), Art. 105 Rn. 23; Georg Hermes, in: Horst Dreier (Hrsg.), Grundgesetz-Kommentar, Tübingen 2003, Art. 69 Rn. 7. 
Bundesländern keine eigenen oder besonderen Amtsbefugnisse. ${ }^{42}$ Deshalb ist es auch falsch, vom „stellvertretenden Ministerpräsidenten“ 43 oder auf der Ebene des Bundes vom „Vizekanzler“44 zu sprechen. Soweit also der Ministerpräsident seine Aufgaben selbst wahrnimmt, ist sein Stellvertreter auf seine sonstigen Aufgaben als Minister beschränkt, denn bei der Stellvertretung der Ministerpräsidenten in Deutschland handelt es sich um keine dauernde, „sondern nur um eine intermittierende Tätigkeit, deren Träger im Voraus bestimmt “ 45 ist. Es handelt sich also um eine „Funktionsstellvertretung“ bei vorübergehender Verhinderung des Ministerpräsidenten. ${ }^{46}$ Roman Herzog verweist deshalb darauf, dass die „Bezeichnung als ständiger Vertreter“ nicht bedeutet, „dass der Stellvertreter ständig in Vertretungsgeschäften tätig ist, sondern nur ständig zur Verfügung steht “ ${ }^{47}$. Konsequenterweise erhält der Stellvertreter auch keine „Urkunde“ über die Bestimmung zum Stellvertreter, wie dies aber für seine Position als Minister der Fall ist. In der Staatspraxis von Rheinland-Pfalz ${ }^{48}$ und den meisten anderen Bundesländern ${ }^{49}$ erhält er „nur“ ein „Bestellungsschreiben“.

\section{Die Unterschiede in den verfassungsrechtlichen Regelungen für die Ernennung zum Minis- ter und die Bestimmung als Stellvertreter des Ministerpräsidenten in Rheinland-Pfalz}

Dass es sich beim Amt des Ministers und bei der Funktion des Stellvertreters um zwei unterschiedliche rechtliche Positionen handelt, wird in Rheinland-Pfalz noch durch weitere Besonderheiten der Landesverfassung deutlich, und zwar unter anderem durch die verschiedenen Standorte der Regelungen und die unterschiedlichen Begriffe für die verschiedenen Akte der Regierungsbildung.

\subsection{Die unterschiedlichen Beteiligungsformen des Landtags bei der Regierungsbildung}

Bei allen drei Schritten der Regierungsbildung (Wahl des Ministerpräsidenten, Ernennung der Minister und Bestimmung des Stellvertreters) wird der Landtag gesondert tätig. Er „wählt" nach Art. 98 Abs. 2 Satz 1 LV-RP mit der so genannten absoluten Mehrheit den Ministerpräsidenten. Die Ernennung der Minister durch den Ministerpräsidenten bedarf

42 Vgl. für die Bundesländer zum Beispiel Volker Epping, a.a.O. (Fn. 15), Art. 29 Rn. 36; Karl Schweiger (Fn. 8), Art. 46 Rn. 2 und für den Bund zum Beispiel Martin Oldiges, in: Michael Sachs (Hrsg.), Grundgesetz-Kommentar, München 2009, Art. 69 Rn. 9/10; Hans-Peter Schneider, in: Rudolf Wassermann (Hrsg.), Alternativkommentar zum Grundgesetz für die Bundesrepublik Deutschland, Neuwied 1984, Art. 69 Rn. 2.

43 Vgl. Karl Schweiger, a.a.O. (Fn. 8), Art. 46 Rn. 2.

44 Vgl. zum Beispiel Martin Oldiges, a.a.O. (Fn. 42), Art. 69 Rn. 9/10, Hans-Peter Schneider, a.a.O. (Fn. 42), Art. 69 Rn. 2.

45 Karl Schweiger, a.a.O. (Fn. 8), Art. 46 Rn. 2.

46 Vgl. Volker Epping, a.a.O. (Fn. 15), Art. 29 Rn. 36; Martin Oldiges, a.a.O. (Fn. 42), Art. 69 Rn. 9/10; Hans-Peter Schneider, a.a.O. (Fn. 42), Art. 69 Rn. 2.

47 Roman Herzog, a.a.O. (Fn. 13), Art. 69 Fn. 1.

48 Nach dem Schreiben der Staatskanzlei Rheinland-Pfalz vom 10. September 2007 an den Autor wird das „Bestellungsschreiben ... durch den Ministerpräsidenten zusammen mit der Ernennungsurkunde vor Zustimmung des Landtags dem betreffenden Staatsminister ausgehändigt“.

49 Ergebnis einer Umfrage des Autors in Zusammenhang mit der Dokumentation „Die Ministerpräsidenten und ihre Stellvertreter 1990 bis 2013“, deren Veröffentlichung für 2014 geplant ist. 
der „ausdrücklichen Bestätigung des Landtags“ (Art. 98 Abs. 2 Satz 3 LV-RP) ${ }^{50}$, während die Bestimmung seines Stellvertreters nur „mit Zustimmung des Landtags“ rechtmäßig ist (Art. 105 Abs. 3 Satz 3 LV-RP). Für diese drei unterschiedlichen Handlungen des Parlamentes werden deshalb auch unterschiedliche Begriffe verwendet, nämlich: wählen, (nachträglich) bestätigen und (ausdrücklich) zustimmen.

Was man unter Zustimmung versteht und welcher Rechtsnatur das Erfordernis der Zustimmung ist, wird weder vom Grundgesetz noch von den Landesverfassungen erläutert. Nach Klaus Stern „hielt man den Begriff ... für geklärt“. Er weist auf das Privatrecht hin und formuliert, dass „die Zustimmung die Wirksamkeitsvoraussetzung für die Rechtshandlung eines anderen Rechtssubjektes" 51 ist. Das bedeutet: Erst durch die Zustimmung des Landtags wird die Bestimmung des Stellvertreters des Ministerpräsidenten rechtswirksam.

\subsection{Die unterschiedlichen Standorte der beiden Regelungen}

Wie schon ausgeführt wird die Bestimmung des Stellvertreters nicht in Art. 98 LV-RP, der die Einzelheiten der sonstigen Regierungsbildung enthält, sondern in Art. 105 LV-RP geregelt. Dies hat rechtlich nicht unbedeutende Folgen. Während der Ministerpräsident für die Entlassung eines Ministers nach Art. 98 Abs. 2 Satz 4 LV-RP die (vorherige) Zustimmung des Landtags benötigt, ist für eine Entpflichtung des Stellvertreters keinerlei vorherige beziehungsweise nachträgliche Beteiligung des Landtags erforderlich. Die Ansicht der Staatskanzlei $^{52}$, dass bei der „Entlassung eines Ministers aus seiner Funktion als stellvertretender Ministerpräsident ein gleich gelagertes Verfahren anzuwenden (actus contrairus) “ sei, ist nicht stichhaltig und steht nicht im Einklang mit der Landesverfassung. Der Verfassungsgeber hat nur für die Entlassung der Minister aus dem Amt ausdrücklich in Art. 98 Abs. 2 Satz 4 LV-RP die Mitwirkung des Landtags geregelt, nicht aber für die Abberufung des Stellvertreters. In Art. 105 Abs. 3 Satz 3 LV-RP wird keine Mitwirkung bei der Entpflichtung eines Ministers von der Funktion als Stellvertreter des Ministerpräsidenten gefordert.

In Bayern liegt eine vergleichbare Regelung vor. Während nach Art. 45 LV-BY sowohl für die Berufung als auch die Entlassung der Minister die Zustimmung des Landtags erforderlich ist, schreibt Art. 46 LV-BY nur für die Bestimmung und nicht für die Abberufung des Stellvertreters des Ministerpräsidenten die parlamentarische Zustimmung vor. Man kann deshalb, wie Karl Schweiger ausführt, höchstens sagen, dass der Landtag indirekt an der Entpflichtung beteiligt wird, denn wenn der Ministerpräsident die Bestimmung seines bisherigen Stellvertreters rückgängig macht, dann muss er unverzüglich einen Nachfolger bestimmen und dem muss der Landtag nach Art. 105 Abs. 2 Satz 3 LV beziehungsweise Art. 46 LV-BY zustimmen. ${ }^{53}$

50 Nach der Einfügung des Art. 98 Abs. 3 LV im Jahre 1991 kann sich die Bestätigung nur noch auf die Minister und nicht die Gesamtregierung beziehen; vgl. Richard Ley, a.a.O. (Fn. 38), S. 363; ders., a.a.O. (Fn. 6), S. $136 \mathrm{ff}$.

51 Klaus Stern, a.a.O. (Fn. 13), \$27 IV 6 d).

52 Schreiben an den Autor vom 11. Juni 2013. Leider wird in diesem Schreiben auch die unzutreffende Bezeichnung „stellvertretende Ministerpräsidentin“ verwendet.

53 Karl Schweiger (Fn. 8), Art. 46 Rn. 3. So im Ergebnis auch Klaus-Eckart Gebauer, a.a.O. (Fn. 29), Art. 98 Rn. 25. 
6.3. Die unterschiedlichen verfassungsrechtlichen Positionen: Die Verfassungsorganqualität der Minister - die Personenbezogenheit der Stellvertretung

Ein weiterer Unterschied zwischen den beiden verfassungsrechtlichen Positionen ist von entscheidender Bedeutung. Mit der nachträglichen Bestätigung des Landtags zur Ernennung zum Minister wird dieser Mitglied des Verfassungsorgans Landesregierung. Durch diese Mitgliedschaft stehen ihm eigene Rechte sowie Pflichten zu, die unter anderem in Art. 104 sowie Art. 105 Abs. 1, Abs. 2 Satz 1 und Abs. 3 LV-RP geregelt sind. Darüber hinaus hat nach der Rechtsprechung des rheinland-pfälzischen Verfassungsgerichtshofes der Minister selbst auch Verfassungsorganqualität. ${ }^{54}$

Über die Rechte und Pflichten des Stellvertreters des Ministerpräsidenten geben die Landesverfassungen und das Grundgesetz jedoch keinerlei Auskunft - mit einer Ausnahme, die jedoch sehr wichtig ist: Der Stellvertreter steht in einem besonderen Verhältnis zum Ministerpräsidenten, der ihn für diese Funktion bestimmt hat. Dies kommt durch die Verwendung des Possessivpronomens („seinen Stellvertreter“) sowohl im Grundgesetz als auch in der Rheinland-Pfälzischen Landesverfassung ${ }^{55}$ und in gleicher oder ähnlicher Formulierung in den meisten anderen Landesverfassungen ${ }^{56}$ zum Ausdruck. Es besteht hinsichtlich seiner Stellung als Stellvertreter des Ministerpräsidenten - nicht bezüglich seiner Stellung als Mitglied der Landesregierung - also „nur“ ein besonderes Verhältnis zwischen dem Ministerpräsidenten und seinem Stellvertreter. Dies wird von Bodo Pieroth deshalb zutreffend als „eine personenbezogene Stellvertretung "57 bezeichnet. ${ }^{58}$

Die Personenbezogenheit soll durch zwei Gegenbeispiele noch verdeutlicht werden. Als einziges Bundesland bestimmt in Bremen nicht der Präsident des Senats den Stellvertreter (Bürgermeister) selbst, sondern dieser wird für die gesamte Wahlperiode vom Senat gewählt (Art. 114 LV-HB). ${ }^{59}$ Dies bedeutete, dass zum Beispiel nach dem Rücktritt von Henning Scherf im Jahre 2005 der bisherige Bürgermeister Thomas Röwekamp auch unter dem neuen Senatspräsidenten Jens Böhrnsen ohne erneute Wahl weiterhin im Amt blieb. Als weiteres Gegenbeispiel soll auf Art. 57 GG hingewiesen werden. Danach ist nicht eine bestimmte Person, sondern der Präsident des Bundesrates im Verhinderungsfall Vertreter des Bundespräsidenten. Man nennt diese Stellvertretung „gesetzlich angeordnete Organ-Personalunion" 60 .

54 Vgl. die Nachweise und die Begründung Richard Ley, Staats- und Verfassungsrecht, in: ders. (Hrsg.), Staats- und Verwaltungsrecht für Rheinland-Pfalz, Baden-Baden 1992, A/Rn. 61; Siegfried Jutzi, Staatsrecht, in: Reinhard Hendler / Friedhelm Hufen / ders. (Hrsg.), Landesrecht RheinlandPfalz, Baden-Baden 2012, \$1 Rn. 81 m.w.N.

55 Art. 69 Abs. 1 GG und Art. 105 Abs. 3 Satz 3 LV-RP sprechen von „seinem Stellvertreter“.

56 Vgl. unter anderem Art. 46 Abs. 2 Satz 2 LV-BW; Art. 46 LV-BY; Art. 43 Satz 2 LV-MV; Art. 52 Abs. 3 Satz 2 LV-NRW; Art. 60 Abs. 4 Satz 2 LV-Sachs; Art. 65 Abs. 3 LV-SAnh; Art. 70 Abs. 3 Satz 2 LV-TH. Art. 26 Abs. 2 Satz 2 LV-SH spricht davon, dass der Ministerpräsident aus dem Kreis der Landesminister „für sich“ einen Vertreter bestellt.

57 Vgl. Bodo Pieroth, in: Hans D. Jarass / ders., Grundgesetz für die Bundesrepublik DeutschlandKommentar, München 2012, Art. 69 Rn. 1.

58 Vgl. auch ähnlich Ute Mager, in: Ingo von Münch / Philip Kunig (Hrsg.), Grundgesetz-Kommentar, München 2012, Art. 69 Rn. 3.

59 Vgl. Heinzgeorg Neumann, a.a.O. (Fn. 23), Art. 114 Rn. 4.

60 Ute Mager, a.a.O. (Fn. 58), Art. 69 Rn. 3. 


\section{Ende des Stellvertreterverhältnisses}

\subsection{Abberufung und Rücktritt des Stellvertreters}

Auch wenn es nicht ausdrücklich in den Landesverfassungen geregelt ist, entspricht dem Recht des Ministerpräsidenten zur Bestimmung seines Stellvertreters das Recht zur Entlassung. ${ }^{61}$ Dies ist auf zwei Wegen möglich ${ }^{62}$ : Der Ministerpräsident entlässt entsprechend Art. 98 Abs. 2 Satz 4 LV-RP, das heißt mit Zustimmung des Landtags, den Minister, den er auch zu seinem Stellvertreter bestimmt hat. In dem Augenblick, in dem die Entlassung als Minister rechtskräftig wird, „erlischt auch die Stellvertreterbefugnis“63, denn der Stellvertreter des Ministerpräsidenten muss ein Minister sein. Der Ministerpräsident kann aber auch seinen Stellvertreter weiterhin im Amt des Ministers belassen und ihm nur die Stellvertreterbefugnis entziehen. ${ }^{64}$ Für diesen zweiten Weg der Abberufung ist keinerlei Mitwirkung des Landtags erforderlich. ${ }^{65}$

Selbstverständlich hat der Stellvertreter auch das Recht des Rücktritts. Auch hier gibt es die zwei Möglichkeiten: Der Minister erklärt nur seinen Rücktritt als Stellvertreter und bleibt weiterhin Mitglied der Landesregierung. ${ }^{66}$ Erklärt der Minister seinen Rücktritt als Minister, so erlischt mit der Annahme des Rücktritts durch den Ministerpräsidenten auch seine Stellvertreterfunktion.

7.2. Beendigung des Stellvertreterverhältnisses durch Beendigung des Amtsverhältnisses des Ministerpräsidenten

Die Personenbezogenheit hat entscheidende Auswirkungen auf die Beendigung des Stellvertreterverhältnisses. Wenn der Ministerpräsident von seinem Amt zurücktritt oder das Amtsverhältnis anderweitig endet (wie zum Beispiel Verlust der Amtsfähigkeit oder Tod), so endet auch automatisch die Funktion des Ministers als Stellvertreter. ${ }^{67}$ Der Stellvertreter ist eben kein „Reserve-Regierungschef“, der bei Amtsende oder Tod dessen Amt übernimmt. ${ }^{68}$ Georg Hermes begründet dies für Art. 69 Abs. 1 GG wie folgt: „... eine Vizekanzlerschaft ohne Kanzler hätte nicht mehr den Charakter einer von dessen Willen abhängigen Stellvertretung, sondern den einer interimistischen Nachfolge ohne Kontrolle und Begrenzung durch den amtierenden Kanzler. "69 Nach Art. 99 Abs. 3 LV-RP ist der Ministerpräsident verpflichtet, nach seinem Rücktritt bis zur Wahl seines Nachfolgers im Amt zu bleiben; deshalb kann

61 Vgl. zum Beispiel Volker Busse, in: Karl Heinrich Friauf / Wolfram Höfling, Berliner Kommentar zum Grundgesetz, Berlin 2007 Art. 69 Rn. 3; Roman Herzog, a.a.O. (Fn. 13), Art. 69 Rn. 12.

62 Für den Bund vgl. ebenda, Art. 69 Rn. 12.

63 Ebenda, Art. 69 Rn. 12.

64 Vgl. auch Volker Busse, a.a.O. (Fn. 61), Art. 69 Rn. 3.

65 Vgl. die Ausführungen in Abschnitt 6.1.

66 Zur Problematik dieses Falles vgl. Klaus-Eckart Gebauer, a.a.O. (Fn. 29), Art. 98 Rn. 25.

67 Vgl. zum Beispiel Volker Epping, a.a.O. (Fn. 15), Art. 29 Rn. 39; Reinhard Bergmann, in: Karl-Heinz Seifert / Dieter Hömig (Hrsg.), Grundgesetz für die Bundesrepublik Deutschland. Taschenkommentar, Baden-Baden 1999, Art. 69 Rn. 1, Hans-Peter Schneider, a.a.O. (Fn. 42), Art. 69 Rn. 3.

68 Vgl. Volker Epping, a.a.O. (Fn. 15), Art. 29 Rn. 39; Martin Oldiges, a.a.O. (Fn. 42), Art. 69 Rn. 13; Reinhard Bergmann, a.a.O. (Fn. 67), Art. 69 Rn. 1.

69 Georg Hermes, a.a.O. (Fn. 41), Art. 69 Rn. 7 - Hervorhebung durch den Autor. 
man davon ausgehen, dass auch sein Stellvertreter bis zu diesem Zeitpunkt geschäftsführend die Stellvertreterbefugnis wahrnehmen muss. ${ }^{70}$

Da es in Rheinland-Pfalz keine dem Art. 69 Abs. 2 GG entsprechende Regelung gibt, ist es jedoch möglich, dass die Minister bei Rücktritt des Ministerpräsidenten im Amt bleiben. Dies bedeutet aber nicht, wie der Landtagspräsident in einer Presseerklärung ausführte ${ }^{71}$, dass der Minister, der vom Vorgänger zu seinem Stellvertreter bestimmt wurde, auch unter dem neuen Ministerpräsidenten ohne erneute Bestimmung diese Funktion beibehält. Wie dargelegt, handelt es sich bei der Stellvertretung nicht um ein Amt, sondern um eine personenbezogene Funktionsbestellung. ${ }^{72}$ Das Amt als Minister verbleibt dem bisherigen Stellvertreter, aber die personenbezogene Funktionszuweisung des zurückgetretenen Ministerpräsidenten - er hat nach Art. 105 Abs. 3 Satz 3 LV-RP „seinen Stellvertreter“ bestimmt - erlischt mit der Wahl seines Nachfolgers.

Während im Bund beim Amtsende oder Tod des Bundeskanzlers der Bundespräsident nach Art. 69 Abs. 3 und 63 Abs. 1 GG tätig werden muss, ist dies in den Bundesländern nicht ausdrücklich geregelt. Nur Bayern hat eine Teilregelung vorgesehen. Nach Art. 46 Abs. 3 Satz 4 LV-BY geht die Vertretung des Landes Bayern auf den Landtagspräsidenten über. Wer die anderen Rechte und Aufgaben des Ministerpräsidenten ausübt, scheint strittig. ${ }^{73}$ Damit die Übergangszeit nicht lange andauern soll, sieht die Bayerische Verfassung vor, dass schon in der nächsten Landtagssitzung gewählt werden muss (Art. 44 Abs. 4 LV-BY) und falls eine Neuwahl innerhalb von vier Wochen nicht zustande kommt, der Landtagspräsident den Landtag auflösen muss (Art. 44 Abs. 5 LV-BY).

In der Staatspraxis einzelner Bundesländer wurde das Problem beim Tod des Ministerpräsidenten dahingehend gelöst, dass der (bisherige) Stellvertreter bis zur Neuwahl des neuen Ministerpräsidenten „amtierte“, so unter anderem im Saarland ${ }^{74}$ und in Berlin. ${ }^{75}$ Ein entsprechendes Vorgehen ${ }^{76}$ - und damit ein Abweichen vom oben genannten Grundsatz dürfte in Rheinland-Pfalz notwendig sein. Da die Landesverfassung keinen Zeitraum vorsieht, in dem ein neuer Ministerpräsident gewählt werden muss und deshalb auch keine Regelung kennt, dass bei Nichteinhaltung der Frist der Landtag aufgelöst wird ${ }^{77}$, kann es eine lange Zeit ohne Ministerpräsidenten geben. Diese Situation wäre nur durch eine Selbstauflösung des Landtags nach Art. 84 LV-RP zu beenden. Deshalb ist es sicher angebracht, dass der ehemalige Stellvertreter amtierend die Geschäfte des Ministerpräsidenten wahrnimmt.

70 Vgl. Klaus-Eckart Gebauer, a.a.O. (Fn. 29), Art. 98 Rn. 25; Theodor Meder, Die Verfassung des Freistaates Bayern - Handkommentar, Stuttgart u.a. 1985, Art. 44 Rn. 3.

71 Vgl. „Eveline Lemke ist stellvertretende Ministerpräsidentin. Landtagspräsident Mertes erklärt, dass die Verfassungslage eindeutig ist", a.a.O. (Fn. 3).

$72 \mathrm{Vgl}$. Ausführungen in den Abschnitten 5.3. und 6.3.

73 Vgl. Karl Schweiger, a.a.O. (Fn. 8), Art. 44 Rn. 6a; Theodor Meder, a.a.O. (Fn. 69), Art. 44 Rn. 3.

74 Ulrich Stelkens, in: RudolfWendt/ Roland Rixecker (Hrsg.), Verfassung des Saarlandes. Kommentar, Saarbrücken 2009, Art. 87 Rn. 19 bemängelt das Fehlen einer entsprechenden Regelung, obwohl im Saarland bei der Änderung des Art. 87 Abs. 3 Satz 1 bereits Fälle vorlagen, dass Ministerpräsidenten im Amt verstarben.

75 Vgl. Richard Ley, a.a.O. (Fn. 38), S. 365, S. 367 f. ; demnächst ders., Die Wahl des Regierenden Bürgermeisters von Berlin 1950 bis 2013 (im Erscheinen).

76 Vgl. auch Volker Epping, a.a.O. (Fn. 15), Art. 29 Rn. 39, Fn. 87.

$77 \mathrm{Zu}$ den Regelungen in den anderen Bundesländern vgl. Richard Ley, a.a.O. (Fn. 18), S. 402, S. $416 \mathrm{ff}$. 


\section{Zwei Fallbeispiele}

\subsection{Regierungswechsel von Rudolf Scharping zu Kurt Beck (Oktober 1994)}

Der rechtliche Teil des Regierungswechsels begann mit dem Schreiben von Ministerpräsident Rudolf Scharping vom 30. September 1994 an den Landtagspräsidenten, dass er mit Ablauf des 15. Oktober 1994 sein Amt als Ministerpräsidenten niederlege. ${ }^{78}$ Die Rechtsfolgen dieses Rücktritts bestanden darin, dass, erstens, der scheidende Ministerpräsident nach dem 1991 eingefügten Art. 98 Abs. 3 LV-RP sein Amt ab dem 16. Oktober bis zur Wahl seines Nachfolgers am 26. Oktober 1994 noch geschäftsführend wahrnehmen musste und, zweitens, Rainer Brüderle nun „nur“ noch geschäftsführender Stellvertreter ${ }^{79}$ des (geschäftsführenden) Ministerpräsidenten Scharping war.

In der Sitzung des Landtags am 26. Oktober 1994 verlas der Landtagspräsident ein weiteres Schreiben des Ministerpräsidenten vom selben $\mathrm{Tag}^{80}$, in dem er mitteilte, dass folgende Minister ihren Rücktritt ihm gegenüber erklärt hätten und er diese angenommen habe: Jeanette Rott (Ministerin für die Gleichstellung von Mann und Frau), Ullrich Galle (Minister für Arbeit, Soziales, Familie und Gesundheit) sowie Karl Schneider (Minister für Landwirtschaft, Weinbau und Forsten). Wegen Fehlens einer Art. 69 Abs. 2 GG entsprechenden Regelung war nun im Augenblick vor der Wahl des neuen Ministerpräsidenten folgender Rechtszustand eingetreten: Neben dem geschäftsführenden Ministerpräsidenten Rudolf Scharping waren die anderen am 21. Mai 1991 ernannten Minister kraft Verfassung - ohne weiteren Rechtsakt - Mitglied der künftigen Landesregierung. ${ }^{81}$ Mit der Wahl und Vereidigung von Kurt Beck ${ }^{82}$ endete dann die Amtszeit von Scharping und die jener drei Minister, die gegenüber dem scheidenden Ministerpräsidenten ihren Rücktritt erklärt hatten. Als drittes war aber auch die Funktion von Rainer Brüderle als Stellvertreter von Ministerpräsident Scharping mit dieser Wahl erloschen. ${ }^{83}$

Im Anschluss an seine Vereidigung teilte der neue Ministerpräsident dem Plenum mit, dass die von ihm „geführte Landesregierung aus acht Ministerinnen und Minister bestehe, die auch bisher Mitglieder der Landesregierung gewesen waren“. Die Aufzählung begann: „Der stellvertretende Ministerpräsident Rainer Brüderle wird der Regierung als Minister für Wirtschaft, Verkehr, Landwirtschaft und Weinbau angehören. " ${ }^{44}$ Dann folgten die Namen der weiteren zwei Frauen und fünf Männer und deren Zuständigkeit. Da die acht Staatsminister bereits nach ihrer Ernennung durch Scharping am 21. Mai 1991 als Minister vereidigt worden waren ${ }^{85}$, unterblieb richtigerweise eine (weitere) Vereidigung. Jedoch beantragte der neu gewählte Ministerpräsident die Bestätigung der Landesregierung nach Art. 98 Abs. 2 Satz 3 LV-RP, die mit der Mehrheit des Landtags sodann vorgenommen wurde. Eine solche Bestätigung der Landesregierung dürfte aber nicht notwendig gewesen sein. Nach der

78 Vgl. LT RP PIPr. 12/90, S. 7170.

79 Vgl. Ausführungen in den Abschnitten 6.3. und 8.2.

80 Vgl. LT RP PIPr. 12/90, S. 7170.

81 Vgl. Richard Ley, a.a.O. (Fn. 6), S. 135 f.; Ausführungen in Abschnitt 4.

82 Vgl. LT RP PIPr. 12/90, S. $7172-7175$.

83 Vgl. Ausführungen in den Abschnitten 6.3. und 8.2.

84 LT RP PIPr. 12/90, S. 7175.

85 Vgl. LT RP PIPr. 12/1, S. 19 f. 
Einfügung des Art. 98 Abs. 3 LV-RP im Jahre 1991 muss Art. 98 Abs. 2 Satz 3 LV-RP dahingehend ausgelegt werden, dass einerseits ein neu gewählter Ministerpräsident nicht mehr der Bestätigung des Landtags bedarf und somit sich diese Bestätigung nur auf die sonstigen Mitglieder der Landesregierung bezieht. ${ }^{86} \mathrm{Da}$ alle acht Minister aber bereits am 21. Mai 1991 vom Landtag ihre Bestätigung erhalten hatten, war eine erneute Bestätigung nicht notwendig. ${ }^{87}$

Es könnte vielleicht folgende Überlegung den Ausschlag gegeben haben, dennoch diese Bestätigung zu veranlassen: Aufgrund der Verkleinerung des Kabinetts erhielten einige Minister neue umfangreichere beziehungsweise erweiterte Zuständigkeitsbereiche. So war zum Beispiel Brüderle im Kabinett Scharping Minister für Wirtschaft und Verkehr und unter Beck nun für Wirtschaft, Verkehr, Landwirtschaft und Weinbau zuständig; Jürgen Zöllner, der bisherige Minister für Wissenschaft und Weiterbildung, erhielt das Ressort für Bildung, Wissenschaft und Weiterbildung. Eine solche Überlegung dürfte jedoch mit Blick auf Art. 105 Abs. 2 Sätze 1 und 2 LV-RP, der die Beteiligung des Landtags bei der Änderung der Zuständigkeiten der einzelnen Minister festlegt, nicht angebracht sein. Die Bestätigung nach Art. 98 Abs. 2 Satz 3 LV-RP bezieht sich nur auf das Ministeramt und nicht die einzelne Zuständigkeit. ${ }^{88}$

Jedoch wurde Art. 105 Abs. 2 Satz 3 LV-RP nicht beachtet. Wohl könnte man in der Mitteilung von Ministerpräsident Beck - „der stellvertretende Ministerpräsident, Herr Staatsminister Brüderle, wird der Regierung als Minister für Wirtschaft, Verkehr, Landwirtschaft und Weinbau angehören" 89 - eine indirekte Mitteilung über die Bestimmung eines, nicht seines, Stellvertreters sehen. Jedoch fehlte die nach der Verfassung erforderliche ausdrückliche Zustimmung des Landtags. Da - wie ausgeführt - die Vertreterfunktion von Brüderle mit der Wahl von Beck kraft Verfassung erloschen war, war es Pflicht des neuen Ministerpräsidenten „seinen Stellvertreter“ mit Zustimmung des Landtags zu bestimmen.

Sieht man in der Aufzählung der Mitglieder des Kabinetts - mit dem Hinweis auf den bisherigen Stellvertreter des Ministerpräsidenten - eine ausdrückliche Bestimmung des eigenen Stellvertreters, so fehlt jedoch die Zustimmung des Landtages nach Art. 105 Abs. 2 Satz 3 LV-RP. Die vorgenommene Bestätigung der Regierung gemäß Art. 98 Abs. 2 Satz 3 LV-RP durch den Landtag umfasst nicht die Zustimmung zur Bestimmung des Stellvertreters nach Art. 105 Abs. 2 Satz 3 LV-RP. Es wäre sicher möglich, die beiden Parlamentsakte - Bestätigung der Minister und Zustimmung zur Bestimmung des Stellvertreters - in einem Beschluss vorzunehmen ${ }^{90}$, jedoch müsste dann in der Tagesordnung darauf hingewiesen werden, dass der Beschluss beide Parlamentsakte umfasst. Wenn dies nicht ausdrücklich geschieht, kann eine Rechtswirksamkeit nicht bejaht werden. ${ }^{91}$

86 Vgl. Richard Ley, a.a.O. (Fn. 6), S. 136.

87 Vgl. ebenda.

88 Vgl. die teils kontroverse Diskussion in anderen Bundesländern bei nur teilweise übereinstimmender Rechtslage: Klaus Braun, a.a.O. (Fn. 23), Art. 46 Rn. 29; Heinzgeorg Neumann, a.a.O. (Fn. 12), Art. 29 Rn. 25.

89 LT RP PIPr. 12/90, S. 7175.

90 So die Staatspraxis in Bayern; vgl. zum Beispiel die Kabinettsbildung unter Horst Seehofer am 27. Oktober 2008, LT BAY PIPr. 17/105, S. 7723, S. 7733. Der Tagesordnungspunkt lautete: „Zustimmung zur Berufung der Kabinettsmitglieder sowie zur Bestimmung der Stellvertreterin / des Stellvertreters des Ministerpräsidenten - Artikel 45 und 46 der Bayerischen Verfassung."

91 Vgl. die Ausführungen in Abschnitt 6.1. 
Das erste Kabinett Beck hatte also in der Zeit vom 26. Oktober 1994 bis 20. Mai 1996 keinen nach der Verfassung notwendigen Stellvertreter des Ministerpräsidenten. Erst mit der Regierungsbildung im Mai 1996 wurde Brüderle mit Zustimmung des Landtags ordnungsgemäß zum Stellvertreter von Ministerpräsident Beck bestimmt. ${ }^{92}$

\subsection{Regierungswechsel von Kurt Beck zu Malu Dreyer (16. Januar 2013)}

Die rechtliche Seite des Regierungswechsels begann in der Landtagssitzung am 16. Januar 2013 mit der Information des Landtagspräsidenten, dass Ministerpräsident Kurt Beck ihm am selben Tag ein Schreiben übergeben habe, in dem er seinen Rücktritt erklärt habe. ${ }^{93}$ Damit war der bisherige Ministerpräsident nur noch geschäftsführend bis zur Wahl seiner Nachfolgerin im Amt ${ }^{94}$, und auch „seine Stellvertreterin“, Eveline Lemke, hatte die Funktion einer geschäftsführenden Stellvertreterin des Ministerpräsidenten bis zum Zeitpunkt der Wahl von Malu Dreyer als neue Ministerpräsidentin. ${ }^{95}$ Darüber hinaus blieben auch alle bisherigen Minister - auch Malu Dreyer - im Amt. ${ }^{96}$

Im Anschluss an die Vereidigung der neuen Ministerpräsidentin wurde die Sitzung für kurze Zeit unterbrochen. ${ }^{97}$ Nach Wiedereröffnung wurde die Sitzung mit dem Tagesordnungspunkt „Ansprache der neu gewählten Ministerpräsidentin und Bekanntgabe der Umbildung der Landesregierung durch die neu gewählte Ministerpräsidentin “ fortgesetzt. Da kein Mitglied des letzten Kabinetts von Kurt Beck, das am 18. Mai 2011 ernannt, vereidigt und vom Landtag bestätigt worden war ${ }^{98}$, zurückgetreten war, musste die neue Ministerpräsidentin nur einen Nachfolger für ihr ehemaliges Ministerium ernennen. Die anderen acht Staatsminister blieben im Amt. Am Ende ihrer ersten Ansprache als Ministerpräsidentin teilte Dreyer dann dem Plenum mit, dass sie Alexander Schweitzer zum Minister für Soziales, Arbeit, Gesundheit und Demografie ernannt habe. ${ }^{99}$ Nach seiner Bestätigung gemäß Art. 98 Abs. 2 Satz 3 LV-RP wurde der neue Minister vereidigt. ${ }^{100}$ Danach schloss der Landtagspräsident die Sitzung. ${ }^{101}$

$\mathrm{Da}$ - wie ausgeführt - mit der Wahl der neuen Ministerpräsidentin auch die Stellvertretung von Eveline Lemke beendet war, hätte nun die neue Ministerpräsidentin „ihre“ Stell-

92 Vgl. LT RP PIPr. 13/1, S. 25 f.

93 Vgl. LT RP PIPr. 16/40, S. 2547.

94 Das LT RP PIPr. 16/40, S. 2547 ff. weist ihn deshalb bei seiner Abschiedsrede auch als „Geschäftsführender Ministerpräsident" aus.

95 Vgl. Ausführungen in den Abschnitt 6.3. und 8.2.

96 Vgl. Richard Ley, a.a.O. (Fn. 6), S. 135 f.; Ausführungen in Abschnitt 3.

97 Vgl. LT RP PIPr. 16/40, S. 2555.

98 Vgl. LT RP PIPr. 16/1, S. 18 ff.

99 Die Ernennung geschah in der Staatskanzlei und zwar während der Unterbrechung der Sitzung des Landtags.

100 Vgl. LT RP PIPr. 16/40, S. 2555 f.

101 Nur am Rande sollte angemerkt werden, dass in den amtlichen Unterlagen eigentlich nirgends Erwähnung findet, dass Dreyer als Sozialministerin zurückgetreten ist. Die Landesverfassung hätte es erlaubt, dass sie dieses Amt auch zusätzlich ausgeübt hätte. Man kann aber annehmen, dass dies indirekt geschehen ist, da sie einen Nachfolger für ihr Ministerium ernannt und vom Landtag hat bestätigen lassen. Es bleiben die Fragen: Hat Dreyer gemäß $\$ 3$ MinG-RP eine Entlassungsurkunde bekommen? Von wem hätte diese unterschrieben werden müssen? 
vertreterin bestimmen müssen. Da dies nicht erfolgt und beantragt worden war, konnte der Landtag auch diesem Vorgang nach Art. 105 Abs. 2 Satz 3 LV-RP nicht zustimmen. Folglich hat auch das erste Kabinett von Ministerpräsidentin Malu Dreyer keine nach den Vorschriften der Verfassung notwendige Stellvertreterin der Ministerpräsidentin.

\section{Eine schwierige Verfassungsbestimmung und die Geschichte ihrer Anwendung}

Die Bestimmung seines Stellvertreters ist eine verfassungsrechtliche Pflicht des Ministerpräsidenten, denn nur dadurch ist gewährleistet, dass in einem Verhinderungsfall alle Aufgaben des Regierungschefs und die Funktionsfähigkeit des wichtigsten Verfassungsorgans der Exekutive gewahrt bleiben. Der Minister, der vom Ministerpräsidenten dafür bestimmt wird, nimmt diese Aufgabe neben seinem Ministeramt nicht als zusätzliches öffentlich-rechtliches Amt, sondern nur als weitere Funktionszuweisung wahr. Die Rheinland-Pfälzische Verfassung hat für diese Funktionszuweisung die Zustimmung des Landtags vorgeschrieben (Art. 105 Abs. 2 Satz 3 LV-RP), das heißt erst mit dem Beschluss des Landtages wird die Bestimmung des Stellvertreters durch den Ministerpräsidenten rechtskräftig. Das Mitwirkungsrecht des Landtags ist - wie auch in Bayern - jedoch nur für die Bestimmung und nicht für die Abberufung des Stellvertreters durch den Ministerpräsidenten vorgeschrieben, anders als bei der Abberufung eines Ministers durch den Ministerpräsidenten (Art. 98 Abs. 2 Satz 4 LVRP). Bei der Abberufung des Stellvertreters ist der Ministerpräsident - da keine ausdrückliche Mitwirkung geregelt ist - völlig frei in seiner Entscheidung.

Wie die Verwendung des Possessivpronomens in fast allen Landesverfassungen und dem Grundgesetz zeigt, ist die Stellvertreterfunktion personenbezogen, das heißt, mit dem Rücktritt des Ministerpräsidenten endet auch die Stellvertreterfunktion. In Rheinland-Pfalz führt dies aber, da es keine dem Art. 69 Abs. 2 GG entsprechende Regelung gibt, zu einer besonderen Konstellation: Beim Rücktritt des Ministerpräsidenten erlischt die Funktion des Stellvertreters des Ministerpräsidenten, dieser kann jedoch weiterhin sein Amt als Minister wahrnehmen. Diese Besonderheit hat in der Vergangenheit bei zwei Regierungswechseln zu Pannen geführt, und auch die derzeitige Ministerpräsidentin hat keinen rechtmäßigen Stellvertreter. Diese Panne könnte aber kurzfristig und ohne große Aufwendungen behoben werden, indem die Ministerpräsidentin die derzeitige vermeintliche Stellvertreterin zu „ihrer" Stellvertreterin bestimmt und dafür die Zustimmung im Landtag beantragt. Für diese ist nach Art. 88 Abs. 2 Satz 1 LV-RP nur die Mehrheit der abgegebenen Stimmen erforderlich - was bei den derzeitigen Mehrheitsverhältnissen ohne Probleme zu erreichen wäre.

Die Regelung des Art. 105 Abs. 2 Satz 3 LV-RP ist, wie die Ausführungen zeigen, eine schwierige Bestimmung, die in jüngster Vergangenheit durch fehlerhafte Auslegung zu den beschriebenen Problemen führte. Es sollte hier aber auch erwähnt werden, dass die Vorschrift in den ersten sechs Wahlperioden (1947 bis 1971) meist gar nicht beachtet worden ist. In den 22 Jahren der Regierungszeit von Ministerpräsident Peter Altmeier wurden nur zwei Minister mit Zustimmung des Landtages zu seinen Stellvertretern bestimmt. Im Rahmen einer Kabinettsumbildung am 7. April 1948 wurde Innenminister Jakob Steffan (SPD) diese Funktion zugewiesen. ${ }^{102}$ Nach dem vorübergehenden Ausscheiden der sozialdemokratischen 
Minister aus der Landesregierung im Oktober 1949 bestimmte Altmeier keinen neuen Stellvertreter. Auch in der 2. und 3. Wahlperiode kam er seiner verfassungsrechtlichen Verpflichtung nicht nach. Erst zu Beginn der 4. Wahlperiode bestimmte er den langjährigen Landwirtschaftsminister Oskar Stübinger (CDU) ordnungsgemäß zu seinem Stellvertreter. ${ }^{103}$ Obwohl am Anfang der 5. und 6. Wahlperiode die gesamte Landesregierung zurücktrat, wurde im Rahmen der Regierungsbildung wieder kein Stellvertreter bestimmt. ${ }^{104}$ Bei der Verabschiedung von Stübinger am 30. April 1968 bedankte sich Altmeier bei seinem scheidenden Weggefährten ganz herzlich „für das oftmalige Wirken als Stellvertreter des Ministerpräsidenten" 105 . Aus dieser Bemerkung könnte man schließen, dass zur damaligen Zeit der Ministerpräsident eventuell nur von Fall zu Fall Minister Stübinger mit der Wahrnehmung seiner Vertretung beauftragte. Seit dem 2. Kabinett von Helmut Kohl am Anfang der 7. Wahlperiode im Jahre 1971 bestimmten die neu gewählten Ministerpräsidenten - mit Ausnahme der beiden behandelten Beispiele - ordnungsgemäß ihre Stellvertreter.

103 Vgl. LT RP PIPr. vom 19. Mai 1959; LT RP Drs. Abt. I Nr. 1, S. 10.

104 Vgl. LT RP PIPr. vom 18. Mai 1963, LT RP Drs. Abt. I Nr. 1, S. 8 ff.; LT RP PIPr. vom 18. Mai 1967, 6/1, S. $9 \mathrm{ff}$.

105 LT RP PIPr. 6/18, S. 595. 